\title{
VALOR PRONÓSTICO DE LOS SIGNOS CLÍNICOS HIPERTERMIA HIPERGLUCEMIA E HIPERTENSIÓN SECUNDARIOS A EVENTOS CEREBROVASCULARES HEMORRÁGICOS. HOSPITAL DOCENTE PADRE BILLINI. ENERO-SEPTIEMBRE 2017
}

\section{Prognostic values of the clinic signs hyperthermia, hyperglycemia and hypertension secondary to hemorrhagic cerebrovascular events in teaching. Hospital Padre Billini. January to september 2017}

\author{
Yordano Montesano Jiménez a , Franz Erich López González ${ }^{\text {b }}$ \\ Víctor Cabrera Pimentel ${ }^{\mathrm{c}}$ y Alene Corvil ${ }^{\mathrm{d}}$
}

Recibido: 6 de noviembre, $2019 \bullet$ Aprobado: 24 de enero, 2020

Cómo citar: Montesano Jiménez Y, López González FE, Cabrera Pimentel V, Corvil A. Valor pronóstico de los signos clínicos hipertermia hiperglucemia e hipertensión secundarios a eventos cerebrovasculares hemorrágicos. Hospital docente Padre Billini. enero-septiembre 2017. cysa [Internet]. 15 de febrero de 2021 [citado 23 de febrero de 2021];5(1):87-5. Disponible en: https://revistas.intec. edu.do/index.php/cisa/article/view/2058

\section{Resumen}

Objetivo: determinar el valor pronóstico de la hipertermia, hiperglucemia e hipertensión, secundario a ECV hemorrágico en los pacientes del Hospital Padre Billini, Santo Domingo, República Dominicana, en el periodo enero-septiembre 2017.

Método: se realizó un estudio descriptivo retrospectivo con una muestra de 50 pacientes. Los datos obtenidos fueron procesados con el sistema Epi-info 7.0.

Resultados: el estudio muestra que, de los 50 expedientes revisados, $56 \%$ (23) fueron masculinos. La edad mediana fue de 62 años. Utilizando el rango de edad $\geq 60$ ańos como valor pronóstico de óbito, se obtuvo un $66.67 \%$ (14) fallecidos frente a $62.07 \%$ (18) pacientes vivos, $(\mathrm{p}=0.7382)$. Un $50 \%$ (8) de los pacientes con valores glucémicos $\geq$ $180 \mathrm{mg} / \mathrm{dl}$ terminaron en defunción intrahospitalarios $(\mathrm{p}=0.0246)$ a diferencia de las demás variables, hiperten-

\footnotetext{
${ }^{a}$ Hospital Padre Billini. Santo Domingo, República Dominicana. ORCID: 0000-0003-29007, Correo-e: yordano.montesano@gmail.com yordano@foundationforpeace.org
}

\begin{abstract}
Objective: establish the prognostic value of hyperthermia, hyperglycemia and hypertension for death secondary to hemorrhagic CVE in patients of the "Hospital Padre Billini" in Santo Domingo, Dominican Republic, January September period in 2017.

Methods: It is a descriptive retrospective study with a sample of 50 patients. The data collected was processed with the system Epi-info 7.0.

Results: The study showed that of 50 records reviewed, 56\% (23) were masculine. The median age was 62 years.

Using the age group of $\geq 60$ years as a prognostic value of death, we get that $66.67 \%$ (14) were deceased and $62.07 \%$ (18) were alive ( $\mathrm{p}=0.7382) .50 \%$ (8) of patients with glycemic values $\geq 180 \mathrm{mg} / \mathrm{dl}$ ended up in Intrahospital death $(\mathrm{p}=0.0246)$, in contrast, the variables of hyper-

\footnotetext{
${ }^{\mathrm{b}}$ Hospital Padre Billini. Correo-e: felg447@gmail.com

c Hospital Padre Billini. Correo-e: victorcabrerapimentel@gmail.com

${ }^{d}$ Hospital Padre Billini. Correo-e: lensocorvil2@gmail.com
} 


\section{Yordano Montesano Jiménez, Franz Erich López González, Víctor Cabrera Pimentel y Alene Corvil}

sión e hiperglucemia, que no demostraron relación estadística significativa.

Conclusión: el estudio demuestra una posible relación pronóstico negativa de los valores glucémicos elevados al momento de ingreso con relación a óbito, así como la predilección demográfica metropolitana, edad avanzada y sexo masculino.

Palabras clave: accidente cerebrovascular; hiperglucemia; hipertensión; hipertermia; hemorragia cerebral.

\section{Introducción}

A nivel mundial, de acuerdo con las estimaciones presentadas por la Organización Mundial de la Salud (OMS), alrededor de 15 millones de personas sufren un evento cerebrovascular (EVC). En el año 2015 se reportaron 6.24 millones de fallecidos por esta causa. ${ }^{1}$ Según el Global Burden of Disease (GBD 2016), 2,838,061 (5.19\%) personas fallecieron por ECV hemorrágico, frente a 2,090,170 (4.92 \%) por isquémico, mostrando una ligera diferencia, inclinada a mayor muerte por ECV hemorrágico.

República Dominicana, en el 2016, contaba con 18,706 casos de eventos cerebrovasculares hemorrágicos. En el 1996 se reportaron 1,513 (4.03\%) defunciones, en tanto que en el 2016 contábamos con $2,905(5.11 \%){ }^{2}$

Si bien es cierto que actualmente existen más de 18 escalas de evaluación, siendo la de Rankin modificado e índice de Barthel las más utilizadas, en su uso se ha evidenciado la relativa baja especificidad de las mismas ya que, aun pacientes exhibiendo pronósticos favorables según las escalas, la mortalidad general es de $45 \%$ con solo un $34 \%$ de los pacientes con una resolución satisfactoria. ${ }^{3}$ tension and hyperglycemia did not show any statistical significance.

Conclusions: the study showed a possible negative prognostic for death relationship between elevated glycemic values at hospitalization as well as living in the city, having advance age and being male.

Keywords: Stroke; Hyperglycemia; Hypertension; hyperthermia; cerebral hemorrhage.

El estudio Investigators in the second Intensive Blood Pressure Reduction in Acute Cerebral Hemorrhage Trial (INTERAC2), demostró que la hiperglucemia tiene un valor predictivo pronóstico negativo en los pacientes con ECV hemorrágico de severidad leve a moderada. Los valores considerados de mal pronósticos oscilan entre 7.9 y $25.0 \mathrm{mmol} / \mathrm{L}$ de glucosa en sangre 4 equivalentes a 135 y $450 \mathrm{mg} / \mathrm{dl}$ respectivamente..$^{5-11}$

\section{Material y métodos}

Se realizó un estudio descriptivo con recolección de datos retrospectivo, con la finalidad de determinar el valor pronóstico de muerte que tienen hipertermia, hiperglucemia e hipertensión secundarias a ECV hemorrágico. En el Hospital Docente Padre Billini, Santo Domingo, Distrito Nacional, República Dominicana.

El periodo de estudio del evento comprende de enero a septiembre del año 2017. Los datos fueron recolectados de mayo a julio del año 2018 y la muestra estuvo constituida por una totalidad de 50 pacientes, los cuales fueron diagnosticados con un primer evento cerebrovascular hemorrágico por clínica y/o tomografía axial computarizada, independientemente del sexo, edad y lugar de residencia 
fueron registrados e incluidas en el estudio.

Los criterios de exclusión utilizados fueron:

- Pacientes con ECV hemorrágico y diagnóstico de hipertensión crónica no controlada.

- Personas con EVC hemorrágico y diagnóstico de diabetes no controlada.

- Paciente con ECV hemorrágico que fueron diagnosticados con una infección concomitante / sospecha clínica de foco infeccioso.

- Pacientes referidos por otros hospitales.

- Pacientes con hemorragia intracraneal traumática.

Ningún paciente fue excluido en el estudio.

Los datos fueron recolectados mediante un formulario diseñado para la captación de informaciones y variables de interés, tales como: edad, sexo, variables pronósticas clínicas presentes y estatus de egreso, utilizando los expedientes clínicos del área del archivo del hospital, se tuvo especial atención a: hoja de ingreso, hoja de signograma, hoja temperatura de enfermería, hoja de glucemia, reporte de hemograma al ingreso y certificación de defunción.

Posterior a su análisis, los resultados se expresaron en tablas de frecuencia absoluta y gráficos de las variables de interés.

\section{Resultados}

Identificamos 50 pacientes de los cuales el $56 \%{ }^{23}$ fueron de sexo masculino. La edad mediana fue de 62 años $(\min .=26$, máx. $=92)$. Tanto en hombres como en mujeres el grupo de mayor frecuencia de casos corresponde a 60-79 ańos seguido del grupo de 40-59 años, con $46 \% 20$ y $30 \%{ }^{15}$, respectivamente. Con una distribución de un $28 \%{ }^{14}$ para el sexo masculino y $18 \%{ }^{9}$ femenino en el intervalo de 60-79 años (tabla 1).

Tabla 1. Distribución de casos de ECV hemorrágico según grupo de edad y sexo. Hospital Padre Billini. Enero-septiembre 2017

\begin{tabular}{l|r|r|r|r|r|r}
\hline \multirow{2}{*}{$\begin{array}{l}\text { Grupo de } \\
\text { edad } \\
\text { (años) }\end{array}$} & \multicolumn{4}{|c|}{ Sexo } & \multicolumn{2}{c}{ Total } \\
\cline { 2 - 6 } & Masculino & Femenino & \multicolumn{2}{c}{} \\
\cline { 2 - 6 } & $\%$ & № & $\%$ & № & $\%$ \\
\hline$<\mathbf{4 0}$ & 2 & 4 & 1 & 2 & 3 & 6 \\
\hline $\mathbf{4 0 - 5 9}$ & 10 & 20 & 5 & 10 & 15 & 30 \\
\hline $\mathbf{6 0 - 7 9}$ & 14 & 28 & 9 & 18 & 23 & 46 \\
\hline$\geq \mathbf{8 0}$ & 2 & 4 & 7 & 14 & 9 & 18 \\
\hline Total & 28 & 56 & 22 & 44 & 50 & 100
\end{tabular}

Fuente: formulario para la recolección de datos de los expedientes de los pacientes con Eventos Cerebrovasculares Hemorrágicos en el Hospital Padre Billini, enero-septiembre 2017. $n=50$.

El $48.98 \%{ }^{24}$ reportó el Distrito Nacional como lugar de residencia; la provincia de Santo Domingo en segundo lugar con $32.65 \%{ }^{16}$. Las personas menores de 60 años residieron en Santo Domingo $52.94 \%{ }^{9}$ mientras que aquellos con más o igual de 60 años se asentaban en el Distrito Nacional 56.13 $\%^{17}$ (tabla 2).

La población estuvo constituida por un $58 \%(29$ personas) que al estado de egreso estaba vivo, en contraparte, un $42 \%{ }^{21}$ en condición de fallecido, de los cuales el $57 \%{ }^{12}$ era de sexo masculino y $42 \% 9$ era femenino. La edad mediana de fallecimiento fue 67 años $(\min .=29$, máx. $=87)$. La media de días de internamiento fue de 7.5 días $(\min .=1, \max .=41)$. 


\section{Yordano Montesano Jiménez, Franz Erich López González, \\ Víctor Cabrera Pimentel y Alene Corvil}

Gráfico 1. Distribución de género según condición vital de egreso. Hospital Padre Billini. Eneroseptiembre 2017

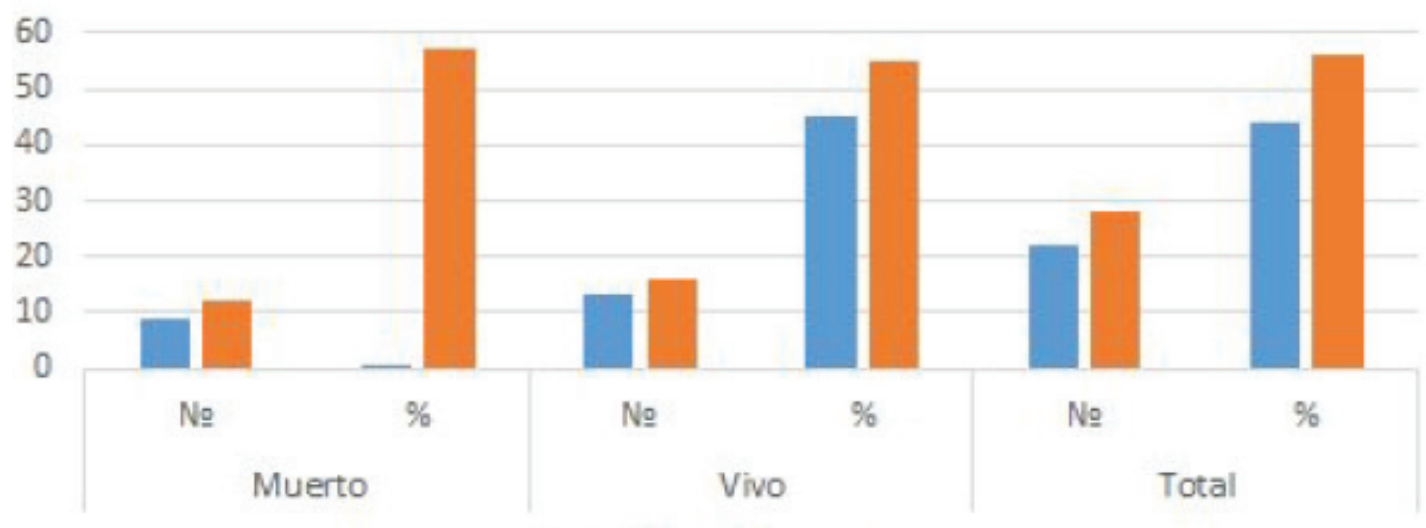

Femenino Masculino

Fuente: formulario para la recolección de datos de los expedientes de los pacientes con Eventos Cerebrovasculares Hemorrágicos en el Hospital Padre Billini, enero-septiembre 2017. N=50.

El $48.89 \%{ }^{20}$ de los pacientes tenía un $50 \%$ de severidad medido, mientras $24.44 \%{ }^{11}$ de los pacientes tenían un $80 \%$ de severidad, según la escala de Hunt-Hess, respectivamente.

De los 50 pacientes incluidos en el estudio, solo 31 tenían un diagnóstico topográfico realizado, donde el $83 \%{ }^{22}$ resultaron ser hemorragias intracraneales de los cuales el $75 \%{ }^{9}$ se reportan como fallecidos al momento de egreso, el $80 \%{ }^{16}$ de los casos tenían ubicación supratentorial, por otro lado, las hemorragias subaracnoideas constituyeron el $16 \%, 5$ del total donde el $25 \%{ }^{3}$ fallecieron en la estadía hospitalaria.

Gráfico 2. Diagnóstico de los pacientes según la condición de egreso. Hospital Padre Billini. Eneroseptiembre 2017

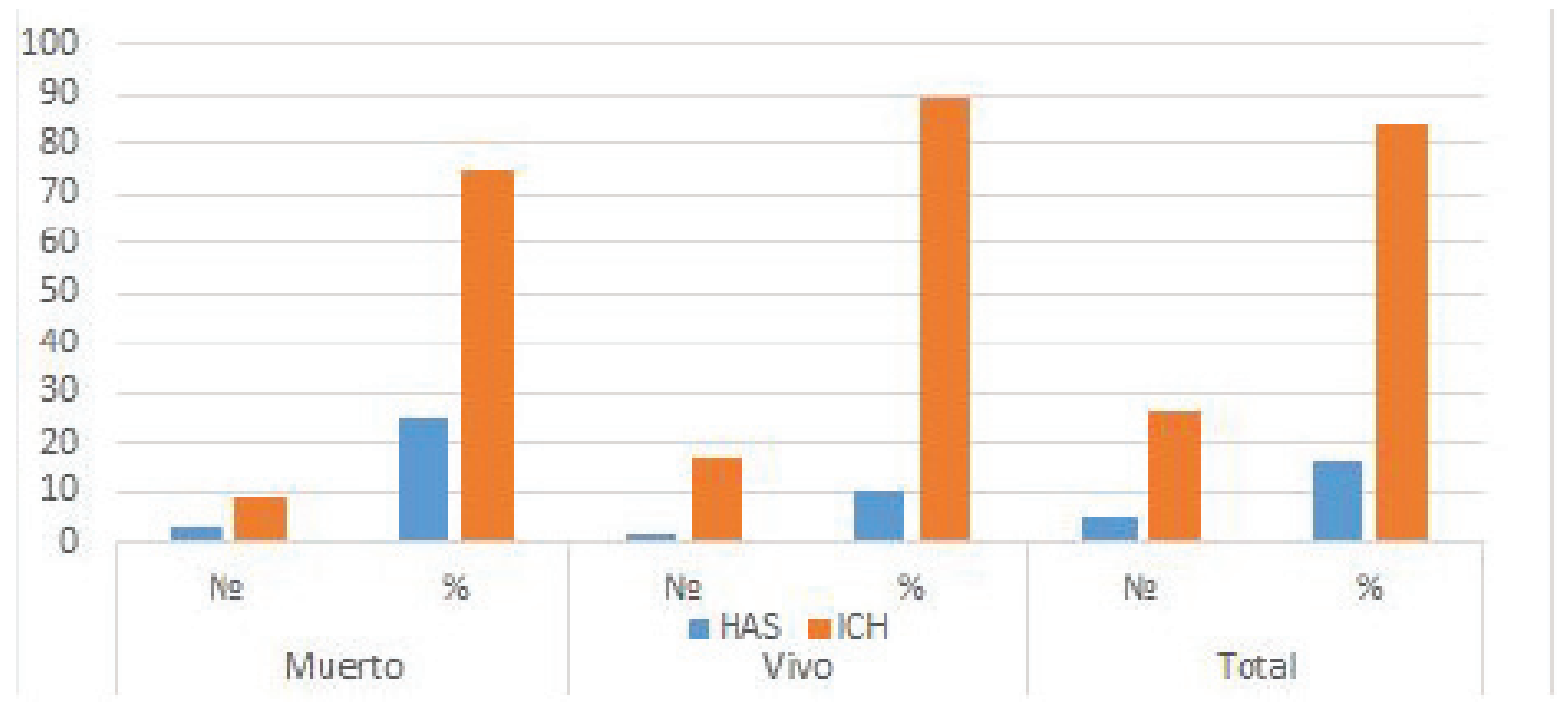

Fuente: formulario para la recolección de datos de los expedientes de los pacientes con Eventos Cerebrovasculares Hemorrágicos en el Hospital Padre Billini, enero-septiembre 2017. n= 31 . 
Con relación a los valores glucémicos al momento de ingreso, se encontró una diferencia pronóstica significativa (Chi2 $=5.05 ; \mathrm{p}=0.0246)$ debido a que el $50 \%{ }^{8}$ de los pacientes que tuvieron hiperglucemia $(\geq 180 \mathrm{mg} / \mathrm{dl})$ al momento de ingreso concluyeron en fallecimientos intrahospitalarios, solo un $17 \% 5$ permaneció con vida al momento de egreso. Sin embargo, no se encontraron diferencias significativas entre los grupos de pacientes mayores y menores a 60 años de edad (p 0.7382). Asimismo, como los que ingresaron con hipertensión ( $\mathrm{p} 0.5744$ ).

Tabla 2. Distribución provincial según grupos de edad. Hospital Padre Billini, enero-septiembre 2017

\begin{tabular}{|c|c|c|c|c|c|c|}
\hline \multirow{3}{*}{ RESIDENCIA } & \multicolumn{4}{|c|}{ Edad } & \multirow{2}{*}{\multicolumn{2}{|c|}{ Total }} \\
\hline & \multicolumn{2}{|c|}{$<60$} & \multicolumn{2}{|c|}{$\geq 60$} & & \\
\hline & № & $\%$ & № & $\%$ & № & $\%$ \\
\hline Azua & 0 & 0 & 2 & 6.25 & 2 & 4.08 \\
\hline Distrito Nacional & 7 & 41.18 & 17 & 53.13 & 24 & 48.98 \\
\hline Hato Mayor & 0 & 0 & 2 & 6.25 & 2 & 4.08 \\
\hline Monte Plata & 0 & 0 & 1 & 3.13 & 1 & 2.04 \\
\hline Peravia & 0 & 0 & 1 & 3.13 & 1 & 2.04 \\
\hline San Cristobal & 1 & 3.13 & 1 & 3.13 & 2 & 4.08 \\
\hline $\begin{array}{l}\text { San fransico de } \\
\text { Macoris }\end{array}$ & 0 & 0 & 1 & 3.13 & 1 & 2.04 \\
\hline Sto Dgo & 9 & 52.94 & 7 & 21.88 & 16 & 32.65 \\
\hline
\end{tabular}

Fuente: formulario para la recolección de datos de los expedientes de los pacientes con Eventos Cerebrovasculares, enero-septiembre 2017. n= 50. El 48.98 \% ${ }^{21}$ reportó Distrito nacional como lugar de residencia, siendo en segundo lugar la provincia de Santo Domingo con un $32.65 \%{ }^{16}$. Las personas menores de 60 años se asentaban en el Distrito Nacional constituyendo $56.13 \%{ }^{17}$.
Tabla 3. Valor pronóstico de la edad, hiperglucemia, hipertensión en pacientes con ECV hemorrágico según la condición vital al egreso. Hospital Padre Billini, enero-septiembre 2017

\begin{tabular}{|c|c|c|c|c|c|c|c|c|}
\hline \multirow{3}{*}{$\begin{array}{c}\text { Variable } \\
\text { pronostico }\end{array}$} & \multicolumn{4}{|c|}{ Egreso } & \multirow{2}{*}{\multicolumn{2}{|c|}{ Total }} & \multirow{3}{*}{$\begin{array}{c}\text { Chi- } \\
\text { cuadrado }\end{array}$} & \multirow{3}{*}{ Valor de $p$} \\
\hline & \multicolumn{2}{|c|}{ Muerto } & \multicolumn{2}{|c|}{ Vivo } & & & & \\
\hline & № & $\%$ & № & $\%$ & № & $\%$ & & \\
\hline Edad $\geq 60$ afios & 14 & 66.67 & 18 & 62.07 & 32 & 64.00 & 0.1117 & 0.7382 \\
\hline $\begin{array}{l}\text { Glucemia } \geq 180 \\
\text { maldl }^{-1}\end{array}$ & 8 & 50 & 5 & 17.86 & 13 & 29.55 & 5.0535 & 0.0246 \\
\hline \begin{tabular}{|l|} 
Hipertension \\
arterial \\
(Sisolica $\geq 140$ o \\
diastolica $\geq 100$ \\
mmHg)
\end{tabular} & 16 & 76.19 & 20 & 68.97 & 36 & 72.00 & 0.3154 & 0.5744 \\
\hline
\end{tabular}

Fuente: formulario para la recolección de datos de los expedientes de los pacientes con Eventos Cerebrovasculares Hemorrágicos en el Hospital Padre Billini, enero-septiembre 2017. Los datos están presentados en n, \% y el valor de $\mathrm{p}$ se aplicó usando chi-cuadrado. $\mathrm{n}=50$.

\section{Discusión}

La resolución del ECV se predice a través de diferentes escalas pronósticas, siendo estas el foco de investigación clínica de interés. Esta investigación describió variables independientes y su posible relación con el valor pronóstico de muerte del cuadro patológico.

La mayoría de los casos fueron residentes de la zona metropolitana. Aunque la edad reportada más frecuente fue la edad avanzada $(\geq 60)$, el vínculo con 


\section{Yordano Montesano Jiménez, Franz Erich López González, Víctor Cabrera Pimentel y Alene Corvil}

el pronóstico de muerte fue incierto, a diferencia de otros estudios donde se demuestra la correlación positiva ${ }^{13}$, este hallazgo podría estar relacionado de la misma manera en la cual se correlacionan enfermedades crónicas propias de áreas metropolitanas, donde existen factores de riesgo para ECV como son: hipertensión arterial, hiperlipidemia, obesidad y diabetes.

De las variables pronósticas estudiadas, se encontró que la hiperglucemia, situación demográfica metropolitana y el sexo masculino son las implicadas en pronóstico negativo, evidenciado por un mayor número de casos fallecidos en comparación con los egresados vivos. Diversos estudios relacionan con diferentes abordajes las variables presentadas y corroboran los hallazgos encontrados en relación con el sexo, así como hiperglucemia y la distribución demográfica. ${ }^{11,15-19}$

Consideramos posible la existencia de una relación entre la severidad del ECV y un previo compromiso endotelial funcional y estructural secundario al estado hipoglucémico. Un estudio de metaanálisis revisó diferentes resultados de regiones y continentes, encontrando que el sexo femenino poseía mayor riesgo de morir, cntrario a la información que se obtuvo en esta investigación. ${ }^{20}$ En contraste con la presente y las consultadas, no se hallaron diferencias significativas de pronóstico de muerte asociado a hipertensión (sistólica $\geq 140 \mathrm{mmHg}$ o diastólica $\geq$ $100 \mathrm{mmHg}$ ) como variables independientes, pero sí existen múltiples fuentes que presentan hallazgos contrarios ${ }^{20}$; consideramos que la explicación yace en la razón fisiológica de la hipertensión secundaria, como un intento de mantener el flujo sanguíneo colateral a las áreas afectadas.

La hipertermia resultó ser una variable insustancial en este estudio, ya que solo se reportó 1 paciente con temperatura registrada de $38^{\circ} \mathrm{C}$ y condición de egreso satisfactoria. En contraposición con las demás investigaciones que presentan a esta variable como valor pronóstico deletéreo, asociado a vasoespasmos recurrentes, diversos estudios corroboran este hallazgo ${ }^{3,4}$. Destacamos que dentro de las limitaciones de la investigación fue consistente la pérdida de hoja de valores térmicos en los historiales clínicos.

En relación con la variable hiperglucemia, se encontraron vastos estudios realizados, además de varios metaanálisis que apoyan la relación pronóstica negativa entre una glucemia elevada (120 a $140 \mathrm{mg} / \mathrm{dL}$ ) al momento de ingreso en los pacientes diabéticos y no diabéticos ${ }^{20}$; muchos otros no pudieron establecer dicha relación, pero demuestran un patrón de recuperación deletéreo o más tortuoso en relación con los pacientes que no tuvieron hiperglucemia al momento de ingreso. La razón de esto se puede deber a que como algunos estudios realizados en animales demuestran, la hiperglucemia aguda en el escenario de un ECV aumenta la acidosis intracelular local, niveles de glutamato, disrupción de la barrera hematoencefálica y tendencia a resangrado, otros demostraron su asociación positiva con el edema circundante y muerte neuronal local lo que podrían ser fundamentales para los hallazgos presentados en esta investigación.

En el caso del presente estudio se encontró una relación directa y significativa entre tener hiperglucemia al momento de ingreso en pacientes con ECV, independientemente de estos tener historia previa o no de diabetes, y un desenlace mortal. De todos modos, otros estudios establecen la necesidad de investigar más la variable a través de estudios de cohortes ampliado, este contraste de resultados está sustentado en que diversos investigadores han concluido que la hiperglucemia es un fenómeno desencadenado por el $\mathrm{ECV}$, más que una variable que tenga efecto sobre él. ${ }^{8,10,23}$

$\mathrm{Si}$ bien es cierto que en este estudio solamente se pudo establecer la relación pronóstico entre la hiperglucemia al momento de ingreso en ECV y riesgo de muerte, existen otros que plantean una relación entre 
las demás variables por lo que es imperante profundizar sobre este fenómeno, especialmente con un enfoque multicéntrico que permita mejorar la apreciación que tenemos sobre estos conocimientos y mejorar el manejo de esta patología, proporcionando mejores herramientas pronósticas, disminuyendo la secuela de los insultos neurológicos posteriores.

\section{Conclusiones}

Se demostró que los valores glucémicos elevados al momento de ingreso suponen significativamente un valor pronóstico negativo, orientando a la detección precoz y manejo oportuno de este hallazgo en todo paciente cursando un ECV. Por otro lado, no se encontró una relación estadística significativa entre los valores tensionales ni los valores térmicos elevados como factor pronóstico, de la misma forma que la edad, sin embargo, los ECV son más frecuentes en personas que habitan en la zona metropolitana. De igual modo, la probabilidad de desenlace mortal en el sexo masculino es más elevada frente al sexo femenino, como variables independientes, manteniéndose esta relación incluso estratificando el sexo con rangos de edades.

\section{Recomendaciones}

Comparando las conclusiones presentadas con las publicaciones internacionales consultadas, no encontramos información sugestiva a conocimientos nuevos, por lo que es necesario continuar realizando estos análisis para establecer mejores abordajes clínicos y metodológicos respecto a los ECV, con el propósito principal de nacionalizar los protocolos e individualizar respectivamente a nuestros rasgos idiosincráticos. Actualmente, la mayoría de las recomendaciones protocolares y terapéuticas son basadas en intervenciones norteamericanas y europeas, y aun siendo información de alto valor científico deben ser aplicadas de forma particular en nuestros centros de salud y disponibilidad de recursos, ya que a través de la historia se han comprobado el efecto diverso sobre las variables étnicas y sociodemográficas.

\section{Bibliografía}

1. Organización Mundial de la Salud. Las 10 principales causas de defunción. [Actualizada enero 2017]. Disponible en: http://www.who. $\mathrm{int} /$ mediacentre/factsheets/fs310/es/. Institute for Health Metrics and Evaluation. Global Burden of Disease StudyData visualization: GBD Compare; 2018.

2. Hansen F. Revisión de escalas de pronóstico para el Accidente Cerebrovascular agudo hemorrágico Revista Memoriza.com 2010;7:32-41. Disponible en: http://www.memoriza.com/docum entos/revista/2010/pronostico_hemorragia_ 7_32-41.pdf

3. Díez Tejedor E, Fuentes B. Acute Care in Stroke: The Importance of Early Intervention to Achieve Better Brain Protection. Cerebrovascular Diseases. 2003;17(1):130-7.

4. Saxena A, Anderson CS, Wang X, Sato S, Arima $\mathrm{H}$, Chan E, Muñoz-Venturelli P, Delcourt C, Robinson T, Stapf C, Lavados PM, Wang J. Prognostic Significance of Hyperglycemia in Acute Intracerebral Hemorrhage: The INTERACT2 Study. Stroke. 2016;47(3):682-8.

5. Sociedad Española de Diabetes. Conversor $\mathrm{mg} / \mathrm{dl}-\mathrm{mmol} / \mathrm{l}$. 2018. Disponible en: http:// www.sediabetes.org/calculadoras/calculadoras/ conversor.aspx

6. Passero S, Ciacci G, Ulivelli M. The influence of diabetes and hyperglycemia on clinical course after intracerebral hemorrhage. Neurology. 2003 Nov 25;61(10):135-6

7. Candelise L, Landi G, Orazio EN, Boccardi E. Prognostic Significance of Hyperglycemia in Acute Stroke. Archives of Neurology. 1985 Jul 1;42(7):661-3. 


\section{Yordano Montesano Jiménez, Franz Erich López González, Víctor Cabrera Pimentel y Alene Corvil}

8. Poppe A, et al. Admission diabetes hyperglycemia predicts worse outcome in stroke patients treated with intravenous thrombolysis.ADA. [Internet]. Vol.41:9. Disponible en: 13. http://care.diabetesjournals.org/co ntent/early/2009/01/08/dc081754. short

9. Kimura K, Iguchi Y, Inoue T, Shibazaki K, Matsumoto N, Kobayashi K, et al. Hyperglycemia independently increases the risk of early death in acute spontaneous intracerebral hemorrhage. Journal of the Neurological Sciences. 2007 Apr 15;255(1):90-4.

10. 10. Capes S., Hunt D., Malmberg K., Pathak P., Gerstein H. Stress hyperglycemia and prognosis of stroke in nondiabetic and diabetic patients.Stroke, 2001;32(10):2426-32. Disponible en: https://www.ahajournals.org/do i/ abs/10.1161/str.32.10.2426

11. Kagansky N, Levy S, Knobler H. The role of hyperglycemia in acute stroke. Arch Neurol, 2001;58(8):1209-12. Doi: 10.1001/archneur. 58.8.1209. PMID: 11493160.

12. Centers for Disease Control and Prevention. Stroke Facts. 2017. Disponible en: https:// www.cdc.gov/stroke/facts.html

13. Eun-Chol Song, Kon Chu, Sang-Wuk Jeong, et al. Hyperglycemia Exacerbates Brain Edema and Perihematomal Cell Death After Intracerebral Hemorrhage. Stroke; 2003.

14. Tapia-Pérez J, Gehring S, Zilke R, Schneider T. Effect of increased glucose levels on short-term outcome in hypertensive spontaneous intracerebral hemorrhage. Clinical Neurology and Neurosurgery. 2014;118:37-43.
15. Guo Xiaoming; Li Helin; Zhang Zhiwen; Li Shouchun; Zhang Lizhi; Zhang Jiajing; Han Guiqing. Hyperglycemia and Mortality Risk in Patients with Primary Intracerebral Hemorrhage: A Meta-Analysis. Molecular Neurobiology: 2016;53(Issue 4):2269-75. Disponible en: https://rd.springer.com/article/10.1007/s12035015-9184-4.

16. Anderson CS, Huang Y, Wang JG, Arima H, Neal B, Peng B, Heeley E,Skulina C, Parsons MW, Kim JS, Tao QL, Li YC, Jiang JD, Tai LW,Zhang JL, Xu E, Cheng Y, Heritier S, Morgenstern LB, Chalmers J; INTERACT Investigators. Intensive blood pressure reduction in acute cerebral haemorrhage trial (INTERACT): a randomised pilot trial. Lancet Neurol. 2008;7:391-9.

17. Kagansky N, Levy S, Knobler H. The Role of Hyperglycemia in Acute Stroke. Arch Neurol. 2001 Aug 1;58(8):1209.

18. Woo D, et al. Effect of untreated hypertension in hemorrhagic stroke. Stroke. 2004;35(7):1703. Epub 2004 May 20.

19. Chan Edward S, Anderson Craig, Wang Xia, Arima Hisatomi, Saxena Anubhav, et al. Early Blood Pressure Lowering Does Not Reduce Growth of Intraventricular Hemorrhage following Acute Intracerebral Hemorrhage: Results of the INTERACT Studies. Cerebrovasc Dis Extra 2016;6:71-5

20. Zivin Justin A. Patología Vascular Cerebral Isquémica. En: Cecil R, Goldman L, Schafer A. Goldman-Cecil Tratado de medicina interna. Barcelona: Elsevier; 2016. 
21. Zivin Justin A. Patología Vascular Cerebral Hemorrágica. En: Cecil R, Goldman L, Schafer A. Goldman-Cecil Tratado de medicina interna. Barcelona: Elsevier; 2016.

22. KN G, MC G, Walker C, LC J. Association of blood pressure, blood glucose, and temperature with neurological outcome after childhood stroke. JAMA Neurol. 2016 Jul 1;73(7):829-35.
23. Wu T, Putaala J, Sharma G, Strbian D, Tatlisumak T, Davis S, et al. Abstract WP346: Persistent Hyperglycemia is Independently Associated With Increased Mortality After Intracerebral Hemorrhage. Stroke. 2017 Feb 1;48(Suppl 1): AWP346 LP-AWP346. 